\title{
Análisis sobre la presencia de depresión, estrés o ansiedad y su relación con el desempeño académico en estudiantes de licenciatura
}

\section{Analysis of the presence of depression, stress or anxiety and its relationship with academic performance in undergraduate students}

\author{
ORTIZ-SÁNCHEZ, Pedro Alfonso Guadal †*', BASAVE-TORRES, Rosy Ilda”, SÁNCHEZ- \\ ITURBE, Patricia Guadalupe" y ORTIZ-Y OJEDA, Pedro T."
}

\author{
Instituto Tecnológico de Mérida, México. \\ "Instituto Tecnológico de Tuxtla Gutiérrez, TecNM, México.
}

ID $1^{\mathrm{er}}$ Autor: Pedro Alfonso Guadal, Ortiz-Sánchez / ORC ID: 0000-0002-2466-1837, Researcher ID Thomson: G-52532019, CVU CONACYT ID: 803273

ID $1^{\text {er }}$ Coautor: Rosy Ilda, Basave-Torres / ORC ID: 0000-0002-7305-3897, Researcher ID Thomson: G-5880-2019, CVU CONACYT ID: 720064

ID $2^{\text {do }}$ Coautor: Patricia Guadalupe, Sánchez-Iturbe / ORC ID: 0000-0001-9245-3725, Researcher ID Thomson: G-61322019

ID $3^{\text {er }}$ Coautor: Pedro T., Ortiz-Y Ojeda / ORC ID: 0000-0002-3796-8504

DOI: $10.35429 / J H S .2020 .11 .4 .34 .41$

Recibido Enero 25, 2020; Aceptado Junio 30, 2020

\begin{abstract}
Resumen
Para implementar medidas correctivas y de apoyo, se desarrolla esta investigación educativa que permite identificar la presencia de niveles riesgosos de depresión, ansiedad y/o estrés y evaluar su posible influencia en los elevados de depresión, ansiedad y/o estrés y evaluar su posible influencia en los elevados
índices de reprobación y deserción que presentan los estudiantes de Ingeniería en índices de reprobación y deserción que presentan los estudiantes de Ingeniería en Sistemas Computacionales e Ingeniería Bioquímica; siendo parte de los objetivos del Tecnológico Nacional de México (TecNM) aplicar estrategias de enseñanza que permitan un alto grado de aprendizaje, menor deserción y un mayor grado de egreso de profesionistas competentes. Se aplicó un muestreo aleatorio estratificado sistemático eligiendo a 262 estudiantes quienes participaron respondiendo encuestas previamente valoradas (alfa de Cronbach entre 0.780 y 0.914) ambas carreras normalmente presentan elevados índices de reprobación y baja eficiencia terminal. Los resultados preliminares muestran altos índices de baja eficiencia teming Los restices de imeros grados, siendo esto previamente desconocido para la mayoría de los estudiantes, ansiedad y depresión presentaron ligeras diferencias entre alumnos iniciales con respecto a los de últimos semestres, sin considerar que estos factores pudieran ser determinantes en su desempeño académico. Los estudiantes coinciden en que estos problemas afectan su desempeño académico, en cuanto a la reprobación y resultan ser factor para causar la deserción escolar. Objetivo: correlacionar los resultados obtenidos en las encuestas aplicadas referidas para conocer el grado de depresión, ansiedad y/o estrés entre los estudiantes de 2 licenciaturas diferentes con su nivel de y/o estrés entre ló esto ne desempeno acestreo aleatorio estratifica a estuditentes de Ingenienía Bioquímico Computacionas. depresión e influencia en su desempeño académico, los datos fueron analizados mediante escalas predeterminadas y la prueba de Chi cuadrado para conocer la correlación entre las variables mencionadas. Contribución. - Se detectaron niveles de ansiedad, estrés y depresión en estudiantes de Ingeniería y su correlación en cuanto a la afectación en su desempeño académico, factores a tomar en consideración para evitar la reprobación y la deserción.
\end{abstract}

Ansiedad, Estrés, Deserción escolar

\begin{abstract}
To implement corrective and supportive measures, this educational research is carried out to identify the presence of risky levels of depression, anxiety and / or stress and to evaluate its possible influence on the high rates of failure and drop out of the students of Systems Engineering Computational and Biochemical Engineering. One of the objectives of National Technological Institute of Mexico (TecNM) is to apply teaching strategies that allow a high degree of learning, less dropout and a higher degree of graduation from competent professionals. Applying a systematic stratified random sampling, 262 students were chosen who participated by answering previously assessed surveys (Cronbach's alpha between 0.78 and 0.914$)$. Both programs usually show high rates of failure and low terminal efficiency. Preliminary results show high stress rates and average anxiety in the first grades, this is previously unknown for most of the students; Anxiety in and depression presented slight differences between freshman students and the
ones of last semesters, without considering that these factors could be determining in their academic performance, the students agree that these problems affect their academic performance, in terms of failure and turn out to be a factor to cause dropout. Objective: to correlate the results obtained in the applied surveys referred to know the degree of depression, anxiety and / or stress among the students of 2 different degrees with their level of academic performance. Methodology.- A stratified random sampling was applied to students of Biochemical Engineering and Computer Systems Engineering. 4 surveys were applied to determine anxiety, stress, depression and influence on their academic performance, the data were stress, depression and influence on their academic performance, the data were analyzed using predetermined scales and the Chi square test to find out the correlation between the mentioned variables. Contribution.- Levels of anxiety, stress and depression were detected in Engineering students and their correlation regarding the affectation in their academic performance, factors to be taken into consideration to avoid failure and desertion.
\end{abstract}

Citación: ORTIZ-SÁNCHEZ, Pedro Alfonso Guadal, BASAVE-TORRES, Rosy Ilda, SÁNCHEZ-ITURBE, Patricia Guadalupe y ORTIZ-Y OJEDA, Pedro T. Análisis sobre la presencia de depresión, estrés o ansiedad y su relación con el desempeño académico en estudiantes de licenciatura. Revista de Educación Superior. 2020. 4-11:34-41.

\footnotetext{
*Correspondencia al Autor (Correo Electrónico: portiz130@ gmail.com)

$\dagger$ Investigador contribuyendo como primer autor.
} 


\section{Introducción}

Según la OMS (Organización Mundial de la Salud) la depresión es un trastorno afectivo y mental frecuente que se caracteriza por la presencia de angustia, pérdida de interés o placer, sentimientos de culpa o ausencia de autoestima. Se calcula que afecta a más de 300 millones de personas en todo el mundo. La ansiedad, la depresión y el estrés son problemas importantes de salud pública en México y a nivel Mundial. Su solución requiere de la labor multidisciplinaria y colaborativa de equipos de salud, capaces de integrar la participación de la sociedad y en particular de las personas afectadas por trastornos depresivos.

A principios de la década se pronosticaba que, en México, en el 2020 la depresión sería la segunda causa de incapacidad de cualquier condición médica (Serrano, Rojas y Ruggero, 2013), estos trastornos son prevalentes en la población joven, al menos el 14.3 por ciento de los ciudadanos padece trastornos de ansiedad generalizada, enfermedad de salud mental más común en el país, le sigue la depresión y adicciones, ambas en un porcentaje de 9 por ciento. El 50\% de los trastornos de ansiedad se presenta antes de los 25 años, por su parte, la depresión presenta la mitad de sus casos entre los 20 y 30 años; el estrés permanece prácticamente en todas y cada una de las etapas de la vida (1).

La frecuencia de trastornos mentales como la ansiedad aumenta en poblaciones con factores de exposición social, ambiental y/o clínicos, su predominio y número difiere según la población en estudio, lo que genera la necesidad de analizar e investigar en poblaciones específicas. Se necesita ampliar esfuerzos que permitan a la sociedad identificarlos, pues son obstáculos al bienestar y la productividad y se deben considerar como retos que se deben superar.

Los estudiantes de nivel superior presentan mayor frecuencia a este tipo de riesgos y constituyen, entre otros, uno de los grupos de mayor interés para su estudio, principalmente porque en este grado de estudios tienen elevados niveles de estrés, exigencias sociales y académicas, que conllevan a desarrollar los trastornos mentales señalados antes (Bohórquez, 2007).
Como factores que afectan el desarrollo académico están la desmedida carga académica, la falta de técnicas correctas de estudio, las evaluaciones y nuevas responsabilidades académicas; también algunos factores no académicos como la falta de apoyo económico o alguna fuente de ingreso, problemas de adaptación a la universidad y a la nueva ciudad, separación de la familia y personales (Benbassat, et al., 2011).

Algunos instrumentos de medición de la ansiedad y la depresión han sido reportados (Joiner et al, 2005; Watson et al, 2007) con diferentes aplicaciones, ventajas y limitantes de acuerdo principalmente al grupo humano que se desee aplicar. Dentro de los instrumentos que se han utilizado para detectar y evaluar la depresión se encuentran también la escala de depresión de Beck, las entrevistas estructuradas y la escala de Zung, siendo esta ampliamente aplicada en investigaciones importantes a nivel internacional y es una de las más empleadas, permite el diagnóstico con relativa certeza y validez en diferentes poblaciones (Diaz et al, 2005), por su amplia aplicación y características específicas la escala de Zung fue seleccionada para esta investigación. El efecto que tienen la ansiedad, el estrés, y la depresión sobre el rendimiento académico ha sido documentado ampliamente, su análisis es objeto de mucho interés porque se ha observado que un desempeño académico pobre induce la reprobación y la deserción escolar, se ha comprobado que cualquier trastorno mental dificulta la toma de decisiones, la retención, el desempeño cognitivo, la memoria a corto y largo plazo, etc. (Leblanc, 2009).

En otros países se han evaluado estudiantes de Medicina, Biología, Fisioterapia, Veterinaria y Zootecnia, Derecho, Odontología e Ingeniería Biomédica en cuanto a la relación en ambas direcciones de la existencia de ansiedad y depresión y el bajo rendimiento y la deserción, considerando que la existencia de uno puede dar origen al otro (González-Olaya et al., 2014; Gutiérrez Rodas et al., 2010); la mayoría de reportes señalan que son los estudiantes de primeros semestres de licenciatura los que muestran mayores índices de depresión, ansiedad y estrés y lo reflejan en bajos rendimientos académicos. 
$\mathrm{Se}$

encontraron

diferentes investigaciones relacionadas con este tema en México, Jiménez Tapia y col. en el 2015 aplicaron la escala CESD-R con muestras no probabilísticas para conocer la prevalencia de depresión en adolescentes en la Cd. de México y en el estado de Michoacán; asimismo, trabajando con estudiantes de nivel medio se correlacionó la existencia de la depresión y el riesgo de fracaso escolar (Hernández-Pozo et al., 2015); en el 2008, se realizó una investigación en estudiantes universitarios para conocer la relación entre el rezago universitario y el estrés (Chapital y Leyva, 2018), por lo anterior se puede afirmar que hay pocos estudios que permiten conocer la presencia de depresión, ansiedad y estrés y su relación con el desempeño académico en estudiantes universitarios de carreras relacionadas con la Ingeniería con marcados niveles de reprobación y deserción, Temas abordados en la presente investigación educativa.

\section{Metodología}

En este proyecto se seleccionaron estudiantes inscritos en las 2 carreras aplicando un muestreo aleatorio estratificado a 262 estudiantes del total, del primero y octavo semestres del periodo enero-junio 2020, los cuales son alumnos de nivel licenciatura en Ingeniería del Tecnológico Nacional de México (TecNM) Campus Tuxtla Gutiérrez y Campus Mérida, el grupo se seleccionó en función a que son los estudiantes de interés para la investigación, el principal objetivo fue conocer el grado de correlación entre la presencia o no de depresión, ansiedad y/o estrés y la percepción del estudiante sobre si estos problemas puedan influir en su desempeño académico y de alguna manera propicien su reprobación y/o deserción, inconvenientes que se presentan con mayor frecuencia en los primeros semestres de la licenciatura, sin embargo, se tomaron en cuenta también a los estudiantes de últimos semestres para comparar la opiniones obtenidas en las encuestas aplicadas.

Para el muestreo se tomó en consideración a la población total de alumnos inscritos en este periodo, la encuesta se les proporcionó con una aplicación móvil desarrollada en esta investigación para este fin.
La respuesta que seleccionó cada encuestado fue almacenada en una base de datos externa alojada en la nube y recuperada para su análisis estadístico. Se aplicaron encuestas independientes de ansiedad, estrés y depresión de un total de 70 preguntas: 20 para ansiedad, 30 para estrés y 20 para depresión, en esta última se utilizó la versión española de la escala de síntomas depresivos de Zung (ESD-Z) obtenido de Lezama (2012), todas las encuestas utilizan una escala de Likert de 4 opciones, la suma de las respuestas a las preguntas produce una puntuación que permite identificar el nivel de depresión $(<50$ puntos para personas que se encuentran en un rango normal, sin depresión, de 50 a 59 puntos para personas que experimentan depresión leve, de 60 a 69 para personas con depresión moderada y $>70$ para personas que experimentan depresión severa, está última es la escala propuesta por Zung (1965), la cual fue adaptada para la escala de Likert.

Las encuestas de ansiedad y estrés fueron evaluadas mediante la propuesta del Melgosa (2011), adaptada y validada por personal docente del TecNM, quienes valoraron el contenido, y aceptaron su aplicación. A manera de ejemplo se observa la evaluación aplicada a Ansiedad:

\begin{tabular}{|l|l|l|l|}
\hline \multicolumn{4}{|c|}{ Variable Ansiedad } \\
\hline $\begin{array}{l}\text { Propuesta de clasificación } \\
\text { basada en escala Likert 4 } \\
\text { puntos adaptada al test de } \\
\text { Melgosa (2011) }\end{array}$ & $\begin{array}{l}\text { Clasificación de la } \\
\text { variable } \\
\text { según test de Melgosa } \\
\text { depresión }\end{array}$ \\
\hline Valoración & Clasificación & Valoración & $\begin{array}{l}\text { Clasifica } \\
\text { ción }\end{array}$ \\
\hline $\begin{array}{l}\text { Si X } \\
\text { (media) } \leq 1\end{array}$ & $\begin{array}{l}\text { Sin } \\
\text { Ansiedad }\end{array}$ & $\begin{array}{l}\text { Si contestó } \\
\text { afirmativo } \\
\text { de } \\
\text { preguntas }\end{array}$ & $\begin{array}{l}\text { Sin } \\
\text { ansiedad }\end{array}$ \\
\hline Si $1<\mathrm{X}<2$ & $\begin{array}{l}\text { Ansiedad } \\
\text { leve }\end{array}$ & $\begin{array}{l}\text { Si contestó } \\
\text { afirmativo } \\
\text { de 5 a 9 }\end{array}$ & $\begin{array}{l}\text { Manifies } \\
\text { ta } \\
\text { propensi } \\
\text { ón a } \\
\text { ansiedad }\end{array}$ \\
\hline Si $2 \geq \mathrm{X} \leq 3$ & $\begin{array}{l}\text { Ansiedad } \\
\text { Moderada }\end{array}$ & $\begin{array}{l}\text { Sí contestó } \\
\text { afirmativo } \\
\text { de 10-13 } \\
\text { preguntas }\end{array}$ & $\begin{array}{l}\text { Tendenc } \\
\text { ia } \\
\text { ansiedad }\end{array}$ \\
\hline Si X $>3$ & $\begin{array}{l}\text { Ansiedad contestó } \\
\text { Severa } \\
\text { afirmativo } \\
\text { más de 14 } \\
\text { preguntas }\end{array}$ & $\begin{array}{l}\text { Tiene } \\
\text { ansiedad }\end{array}$ \\
\hline
\end{tabular}

Tabla 1 Clasificación de la variable Ansiedad 
También fue elaborada una última encuesta de 10 preguntas, se aplicó después de los test señalados y en ella que se cuestionó si la presencia o no de alguno de esos trastornos podía influir en su desempeño académico. Fue necesario concientizar a los estudiantes en cuanto a la importancia de este estudio y se les pidió que señalaran en una escala de valores 1 a 5 (de menos a más) en cada pregunta. La encuesta presenta buen índice de fiabilidad (alfa de Cronbach igual a 0.85) obtenido de una prueba preliminar de aplicación; el cuestionario es una escala de autopercepción de la presencia de depresión, estrés o ansiedad y su relación con el ejercicio escolar.

Se cuestionó en forma clara si la depresión, la ansiedad o el estrés le impiden estudiar, son motivo de bajas calificaciones y si desertar sería o no un recurso que aplicaría si presentara alguna de esas condiciones de salud mental.

La valoración de los test consistió en determinar los valores promedio de las respuestas dadas (Tabla 2).

Variable grado de afectación académica

\begin{tabular}{|l|l|}
\hline \multicolumn{2}{|l|}{$\begin{array}{l}\text { Propuesta de clasificación basada en escala Likert } \\
\text { adaptada al test Afectación Académica }\end{array}$} \\
\hline $\begin{array}{l}\text { Valoración } \\
\mathrm{X} \text { (media) }\end{array}$ & Clasificación \\
\hline Si $1 \leq \mathrm{X}<2$ & Sin Afectación \\
\hline Si $2 \leq \mathrm{X}<$ & Afectación Leve \\
3 & \\
\hline Si $3 \leq \mathrm{X}<4$ & Afectación Moderada \\
\hline Si $\mathrm{X}>4$ & Afectación Severa \\
\hline
\end{tabular}

Tabla 2 Clasificación de la variable Grado de Afectación Académica

Posteriormente fueron procesados y analizados con el software SPSS v.15, realizando de manera inicial un análisis estadístico con alcance descriptivo, se compararon grupos de primero semestres versus grupos de los últimos semestres en ambas carreras. Para conocer la relación de dependencia o no de las variables (ansiedad, estrés y depresión vs encuesta de autopercepción de su influencia en el desempeño escolar) se utilizó la prueba de Chi cuadrada (SPSS v. 15) con un nivel de confianza del $95 \%$ comparándose los resultados de ambas carreras y los obtenidos de los primeros y últimos semestres.
Se realizó un análisis bivariado de acuerdo con la naturaleza de las variables estudiadas, así fue posible conocer aspectos poco conocidos como la prevalencia de la ansiedad, estrés y depresión en los estudiantes y un acercamiento para conocer la opinión sobre la influencia en su desarrollo académico y la posibilidad de la reprobación y/o deserción.

\section{Resultados}

Se evaluaron los resultados de 262 encuestas, los test de ansiedad y estrés (Melgosa, 2011), depresión (Zung, 1965) y el Test de grado de afectación académica propuesto en alumnos de ISC e IBQ, carreras que comparten la característica de ser consideradas como de las de mayor exigencia académica y personal en la Institución y son referidas en forma general por los estudiantes como de gran interés y altamente estresantes. Se observó un menor porcentaje de mujeres participantes (26\%), la media de la edad de los participantes de los primeros semestres fue de 19.4 años y los de últimos semestres 23 años (Gráfico 1).

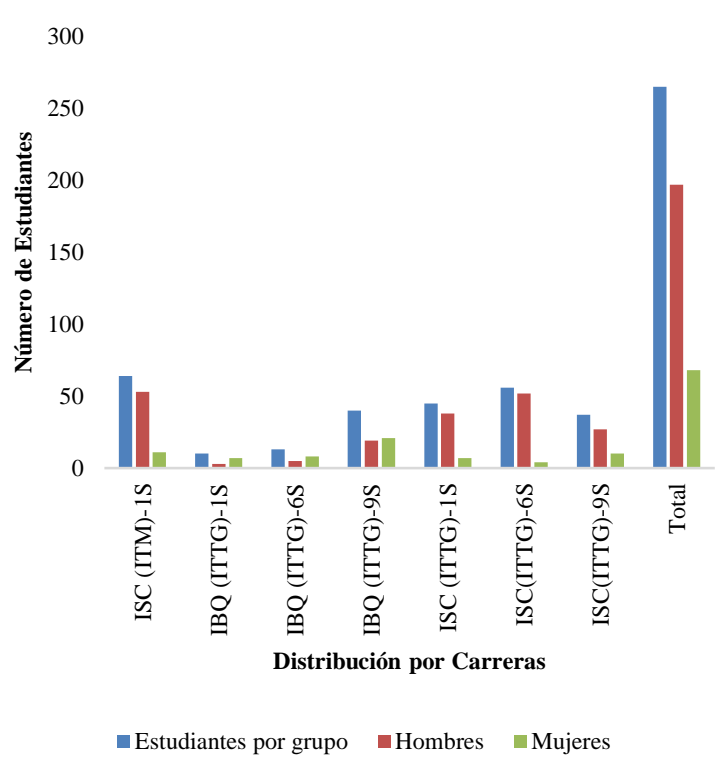

Gráfico 1 Características sociodemográficas generales de los estudiantes

Fuente: Elaboración propia

Los resultados de las pruebas de ansiedad, estrés y depresión se muestran en la Tabla 3. Los resultados permiten observar que la mayoría de los estudiantes no presenta ansiedad (74\%), la autopercepción de ansiedad leve fue alcanzada por un $19.08 \%$ de estudiantes y 17 participantes $(6.49 \%)$ muestran ansiedad severa. 
Al comparar el porcentaje de autopercepción de este factor a niveles de importancia clínica al inicio y al final de la carrera, en IBQ disminuyó del 20\% al 5.66\%, marcando una diferencia significativa, en ISC no hubo gran diferencia siendo $5.56 \%$ y $6.42 \%$ respectivamente.

\begin{tabular}{|lr|c|}
\hline & $\begin{array}{c}\text { Número de casos } \\
\text { observados }\end{array}$ & $\begin{array}{c}\text { Porcentaje } \\
\text { de casos } \\
\text { observados } \\
\text { Escala } \\
\text { Melgosa }\end{array}$ \\
$\begin{array}{l}\text { Resultado de } \\
\text { toda la muestra }\end{array}$ & Escala Melgosa & $\begin{array}{c}\text { Mosad } \\
\text { Sin Ansiedad }\end{array}$ \\
\hline $\begin{array}{l}\text { Con Ansiedad } \\
\text { Moderada }\end{array}$ & 195 & $74.43 \%$ \\
\hline $\begin{array}{l}\text { Con Ansiedad } \\
\text { Severa }\end{array}$ & 50 & $19.08 \%$ \\
\hline Total & 17 & $6.49 \%$ \\
\hline
\end{tabular}

Tabla 3 Casos de ansiedad en la Muestra total IBQ e ISC

Fuente: Elaboración propia

En la Tabla 4 se encuentran los datos obtenidos de la variable estrés para el total de la muestra(sin estrés, con estrés moderado, con estrés severo), la mayoría de los alumnos presentaron estrés moderado y severo, problema que al inicio de la investigación, por las características de la población se supuso que se presentaría, otro factor que pudo influir en las respuestas de los estudiantes fue la contingencia sanitaria por la que atraviesa el país que generó cierta incertidumbre en la población en general.

\begin{tabular}{|l|r|r|}
\hline & $\begin{array}{c}\text { Número de } \\
\text { casos } \\
\text { observados } \\
\text { Escala } \\
\text { Mesultado de } \\
\text { toda la } \\
\text { muestra }\end{array}$ & $\begin{array}{c}\text { Porcentaje de } \\
\text { casos } \\
\text { observados } \\
\text { Escala Melgosa }\end{array}$ \\
\hline $\begin{array}{l}\text { Sin Estrés } \\
\text { Con Estrés } \\
\text { Moderado }\end{array}$ & 2 & $0.76 \%$ \\
\hline $\begin{array}{l}\text { Con Estrés } \\
\text { Severo }\end{array}$ & 44 & $16.79 \%$ \\
\hline Total & 216 & $82.44 \%$ \\
\hline
\end{tabular}

Tabla 4 Casos de estrés en la muestra Fuente: Elaboración propia
En forma particular, los estudiantes de IBQ de los últimos semestres a diferencia de los primeros semestres detectaron estrés moderado (26.42\% vs $30 \%$ ) y también severo (73.58\% vs $70 \%)$; en el caso de ISC los valores para estrés moderado (21.1\% vs $22.02 \%)$ y severo $(77.06 \%$ vs $76.15 \%)$ son parecidos entre los primeros y últimos semestres, sin diferencia significativa en ninguno de los casos; en lo referente a estrés moderado los valores son cercanos a los reportados en otros estudios como el de la Universidad de Caldas (24.6\%), del CES de Medellin (29\%) (Bermudez et al., 2006; Montoya et al., 2010), no así en la percepción de estrés severo. Establecer estas comparaciones hechas para estudiantes universitarios debe permitir enfocar la atención al manejo de los jóvenes para su apoyo y ubicación, ya que, a falta de datos disponibles, se reconoce que se trata de otras situaciones de estudio, contextos distintos y diseños de estudio diferentes.

Se muestran los resultados de los casos de la variable depresión (sin depresión, con depresión leve, con depresión moderada y con depresión severa) para el total de la muestra, ver la tabla 5, el $86 \%$ de estudiantes no presentan depresión, aproximadamente el $12 \%$ depresión leve y se percibe a 2 estudiantes con depresión severa.

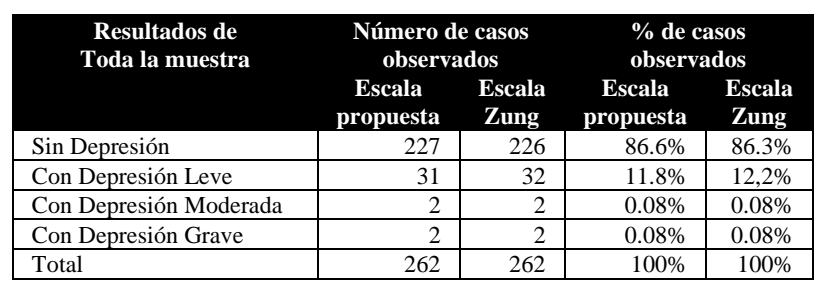

Tabla 5 Casos de Depresión en la muestra total IBQ e ISC

Fuente: Elaboración propia

En la percepción de depresión leve en los estudiantes de Ingeniería Bioquímica existió una tendencia a la disminución del porcentaje promedio entre los primeros y los últimos semestres (de $50 \%$ a 21\%), ninguno mostró depresión severa. En jóvenes de primer semestre de Ingeniería en sistemas computacionales el $86 \%$ no mostraron depresión, el $11 \%$ tuvieron depresión leve, y hay un caso $(0.9 \%)$ con depresión moderada y dos $(1.8 \%)$ con depresión severa y en el último semestre aumentan los jóvenes sin depresión al $94 \%, 4,4 \%$ con depresión leve y un caso $(0.9 \%)$ con depresión moderada. 
Estudios similares (Martín-Monzon (2007); Backovic et al., (2012) aplicados a estudiantes de medicina, licenciatura considerada también altamente estresante, encontraron mayor prevalencia en el estrés entre los primeros y últimos grados, en esta investigación se encontró una tendencia similar para IBQ en ansiedad y depresión, y para ISC únicamente en la depresión; los alumnos de ambas licenciaturas, tanto en el primero como en el último semestre, muestran elevados niveles de estrés, sin diferencias significativas entre sí, esto debe ser tomado en consideración por su importancia en la posible generación de ansiedad y/o depresión y para el apoyo necesario a las personas con depresión severa. Además de la detección de los problemas anteriores y mediante la aplicación de la encuesta de percepción de la afectación en el desempeño académico (ver Tabla 6), los resultados obtenidos visualizan que la ansiedad y el estrés están asociados al bajo rendimiento escolar en más de la mitad de la muestra, los datos señalan que estos problemas afectan moderada y severamente a los estudiantes, particularmente el estrés fue detectado en más del $80 \%$ de los casos como el factor que afecta severamente el desempeño escolar, la percepción fue que la depresión es un factor que les afecta levemente $(12 \%)$.

\begin{tabular}{|l|r|r|r|}
\hline \multirow{2}{*}{ Autopercepción de la afectación } & \multicolumn{3}{c|}{ Total de casos (\%) } \\
\cline { 2 - 4 } & Ansiedad & \multicolumn{1}{c|}{ Estrés } & \multicolumn{1}{c|}{ Depresión } \\
\hline Sin Afectación & $195(74 \%)$ & $2(0.7 \%)$ & $226(86 \%)$ \\
\hline Afectación Leve & 0 & 0 & $32(12 \%)$ \\
\hline Afectación Moderada & $50(19 \%)$ & $44(17 \%)$ & $2(0.7 \%)$ \\
\hline Afectación Severa & $17(6 \%)$ & $216(82 \%)$ & $2(0.7 \%)$ \\
\hline Total & 262 & 262 & 262 \\
\hline
\end{tabular}

Tabla 6 Resultados de la percepción de la afectación en el desempeño académico en la muestra

Fuente: Elaboración propia

Para evaluar la relación existente entre la ansiedad, el estrés y la depresión y la afectación que pudieran causar entre los estudiantes se utilizó la prueba de Chi cuadrada de tendencia, encontrándose en todos los casos una elevada correlación, entendiéndose como una alta frecuencia de reconocimiento, siendo de 0.9998 para ansiedad/afectación académica; 0.9999 para estrés/afectación académica y de 0.9981 para depresión/afectación académica. Se realizó un análisis comparativo por medio de software para identificar los casos en los que se tiene mismo nivel de afectación de variable (ansiedad, depresión o estrés) con relación a la variable afectación académica (ver Tabla 7).

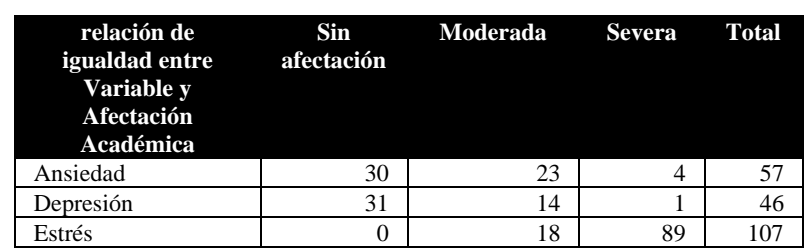

Tabla 7 Casos de igualdad entre variables y afectación académica

Fuente: Elaboración propia

Se encontraron 57 casos donde el nivel de afectación en Ansiedad es igual al nivel de Afectación Académica. Esto es si la variable Ansiedad tiene nivel 1 en afectación también la variable Afectación Académica tiene el mismo nivel y así para los siguientes niveles. De estos 57 casos, 23 casos tienen ansiedad moderada y también presentan una afectación académica moderada; 4 casos tienen ansiedad severa y también presentan una afectación académica severa.

En depresión fueron 46 casos, 14 casos tienen depresión moderada y uno con afectación severa; en donde hubo mayor número de casos fue para el estrés, con 107 casos, 18 con afectación moderada y 89 con afectación severa al desempeño. Se compararon estas pruebas con el nivel de percepción de la afectación para buscar las estrategias para asegurar el bienestar y la salud mental en estudiantes de Ingeniería lo cual podría permitir alcanzar un mejor rendimiento académico y tomando en consideración que tanto la depresión como la ansiedad pueden ejercer daño en las funciones del conocimiento, de la concentración y de la toma de decisiones, deben interpretarse adecuadamente los resultados para el posible auxilio de las necesidades actuales de los estudiantes.

En conclusión, este estudio permite señalar que existe la presencia de altos niveles de estrés, algunos casos de ansiedad e incluso depresión moderada y severa entre los estudiantes de la Institución y además que existe una alta frecuencia de reconocimiento por parte de los estudiantes que la existencia de estos problemas afecta a su desarrollo académico posibilitando su reprobación y su deserción, es de reconocer que en ambos casos no se trató de la misma cohorte, ya que el diseño de experimentos fue el detectar la prevalencia de estos problemas y su relación en la afectación hacia su trabajo académico, lo que resulta de gran interés como Institución educativa por las razones señaladas anteriormente.

ORTIZ-SÁNCHEZ, Pedro Alfonso Guadal, BASAVE-TORRES, Rosy Ilda, SÁNCHEZ-ITURBE, Patricia Guadalupe y ORTIZ-Y OJEDA, Pedro T Análisis sobre la presencia de depresión, estrés o ansiedad y su relación con el desempeño académico en estudiantes de licenciatura. Revista de Educación Superior. 2020 


\section{Referencias}

(1)http://comunicacion.senado.gob.mx/index.ph p/informacion/boletines/39699-14-3-de-lapoblacion-mexicana-padece-trastornos-deansiedad.html. Consultado el 1 de mayo 2020.

Backovic D.V., Zimojinovic J.I., Maksimovic M.(2012) Gender differences in academic stress and burnout among medical students in final years of education. Psychiatr Danub. 24:175181.

Benbassat J., Baumal R., Chan S., Nirel N. (2011) Sources of distress during medical training and clinical practice: suggestions for reducing their impact. Med Teach. 33:486-90.

S. B., Durán M. N., Escobar C., Morales A., Monroy S. A. Ramírez A. (2006) Evaluación de la relación entre rendimiento académico y estrés en estudiantes de medicina. MedUNAB, 9:198-205.

Bohórquez A. (2007). Prevalencia de depresión y ansiedad según las escalas de Zung y evaluación de la asociación con el desempeño académico en los estudiantes de medicina de la Pontificia Universidad Javeriana. Bogotá: Pontificia Universidad Javeriana.

Diaz L. A., Campo A., Rueda G. A., Barros J. A . (2005) Propuesta de una versión abreviada de la escal Zung para depresión.Colomb Med. 36:168-72.

González-Olaya H.L., Delgado-Rico H., Escobar-Sánchez M.,Cárdenas-Ángelone M.A. (2014) Asociación entre el estrés, el riesgo de depresión y el rendimiento académico en estudiantes de los primeros semestres de un programa colombiano de medicina. Fundación educación médica. 17(1):47-54.

Gutiérrez Rodas J. A., Montoya Vélez L. P. Toro Isaza B. E., Briñón Zapata M. A., Rosas Restrepo E., y Salazar Quintero L. E. (2010). Depresión en estudiantes universitarios y su asociación con el estrés académico. CES Medicina, 24(1), 7-17. Recuperado Mayo 07, 2020, en http://www.scielo.org.co/scielo.php?script=sci_ arttext\&pid=S0120-

$87052010000100002 \& \operatorname{lng}=$ en\&tlng=es.
Hernández-Pozo M., Ramírez-Guerrero N., López-Cárdenas S. y Macías-Martínez D.(2015). Relación entre ansiedad, desempeño y riesgo de deserción en aspirantes a bachillerato. Psychologia: avances de la disciplina, 9(1):45-57.

Jiménez Tapia A., Wagner F., Rivera Heredia M. A. y González-Fortaleza C. (2015). Estudio de la depresión en estudiantes de la ciudad de México y del estado de Michoacán por medio de la versión revisada de la CES-D. Salud Mental 38(2):103-107.

Joiner T. E. Jr; Walker R. L.,Pettit J. W., Pérez M. y Cukrowicz K. C (2015). Evidence-based assessment of depression in adults. Psychological assessment. 17:267-277.

LeBlanc V.R (2009) The effects of acute stress on performance:implications for health professions education. Acad. Med. 84(Suppl). Pp.25-33

Lezama S. (2012) Propiedades psicométricas de la escala de Zung para síntomas depresivos en población adolecente escolarizada colombiana. Psychologia avances de la disciplina vol6 No. 1: 91-101.

Martín-Monzon I. M. (2007).Estrés académico en estudiantes universitarios. Apuntes de Psicología. 25:87-99.

Melgosa J. Mente positiva Guía práctica para cualquier situación. Editorial Safeliz S. L. 2011, Madrid, España. Pag 8 y 16.

Montoya L. M., Gutiérrez J. A. Toro B. E.,Briñon M. A., Rosas E., Salazar L. E.(2010) Depresión en estudiantes universitarios y su relación con el estrés académico.RevCES Med 24:7-17.

Zung W.(1965) Self-report depression scale. Archives of General psychiatry 12, 63-70

Serrano Barquín, C., y Rojas García A., y Ruggero C. (2013). Depresión, ansiedad y rendimiento académico en estudiantes universitarios. Revista Intercontinental de Psicología y Educación, 15 (1), 47-60. [Fecha de consulta 21 de mayo de 2020]. ISSN: 01877690. Disponible en: https://www.redalyc.org/articulo.oa?id=802 /80225697004 
Watson D., O’Hara M. W., Simms L. J., Kotov R., Chimielewski M., McDade-Montez E. A.., Gamez W. y Stuart S. (2007). Development and validation of the inventory of depression and anxiety symptoms (IDAS). Psychological assessment. 19:253-268. 